\title{
Correlative Microscopy using Serial Blockface Scanning EM
}

\author{
Thomas Deerinck $^{1}$, Eric Bushong ${ }^{1}$ and Mark Ellisman ${ }^{1,2}$ \\ ${ }^{1}$ The National Center for Microscopy and Imaging Research and the Center for Research \\ in Biological Systems, ${ }^{2}$ Department of Neurosciences, University of California, San \\ Diego, La Jolla, CA, USA.
}

Correlative microscopy aims to combine observations made with disparate techniques, most commonly light and electron microscopy (referred to as CLEM), with the goal of combining the strengths of each imaging approach. For light microscopy, it is the ability to chronicle dynamic cellular processes in living systems or to visualize multiple specific labels over large fields of view, while the electron microscope offers the spatial resolution needed to determine precise subcellular distributions in the context of cellular ultrastructure.

The technique of serial blockface scanning EM (SBEM) is revolutionizing biological electron microscopy by offering unprecedented 3-D views of whole cells and tissues at near nanometer-scale resolution [1]. SBEM employs a miniature ultramicrotome fitted inside an SEM, and instead of imaging sections, it uses backscattered electrons (BSEs) to image the blockface in between repetitive cutting cycles that remove a thin layer of material from the block surface. Continued advancements in instrumentation and specimen preparation protocols have improved the spatial resolution achievable by SBEM such that most organelles and cellular constituents can readily be resolved, making it an ideal approach for CLEM. Specimen preparation for SBEM differs from conventional TEM in that the sample, whether it is a cell culture monolayer or complex tissue, requires much more substantial heavy metal staining than is normally employed for TEM [2]. The intense metal staining reduces specimen charging and improves the BSE yield and image resolution. However, this staining process renders most tissues completely opaque to light, requiring special procedures such as the use of reference fiducial markers and/or X-ray microscopy in order to track and image a precise subregion of a sample by SBEM [3].

SBEM also places several constraints on the labeling approaches that can be used, since the label must be introduced into a bulk specimen. We have focused our efforts on developing molecular-genetic and chemical-labeling approaches designed to facilitate both SBEM and CLEM. These include the tetracysteine/biarsenical labeling system [4], the recombinant fluorescent protein MiniSOG [5], APEX2 (a small and versatile genetically modified ascorbate peroxidase [6]), and a variety of chemical labels that can be used for fluorescence photooxidation of diaminobenzidine. The localization of proteins, macromolecules and organelles using these probes have a number of key advantages over other methods including: 1) excellent preservation of cell ultrastructure, since conventional EM fixation methods can be employed and no permeablizing detergents such as Triton or saponin are required; 2) uniform 3-D labeling can be achieved throughout relatively large volumes of tissue for 3-D light 
and EM; 3) high-resolution labeling. Recent developments with these probes and others will be discussed in the context of their application towards SBEM and CLEM.

\section{References:}

[1] W Denk and H Horstmann PLoS Biol. 2004 Nov; 2(11).

[2] TJ Deerinck et al, Microsc. Microanal. 201016 (suppl 2), 1138-1139.

[3] EA Bushong et al, Microsc Microanal. 2014 Nov 13:1-8.

[4] G Gaietta et al, Science 2002 Apr 19;296 (5567):503-7.

[5] X Shu et al, PLoS Biol. 2011 Apr; 9(4):e1001041.

[6] SS Lam et al, Nat Methods. 2015 Jan;12(1):51-4.

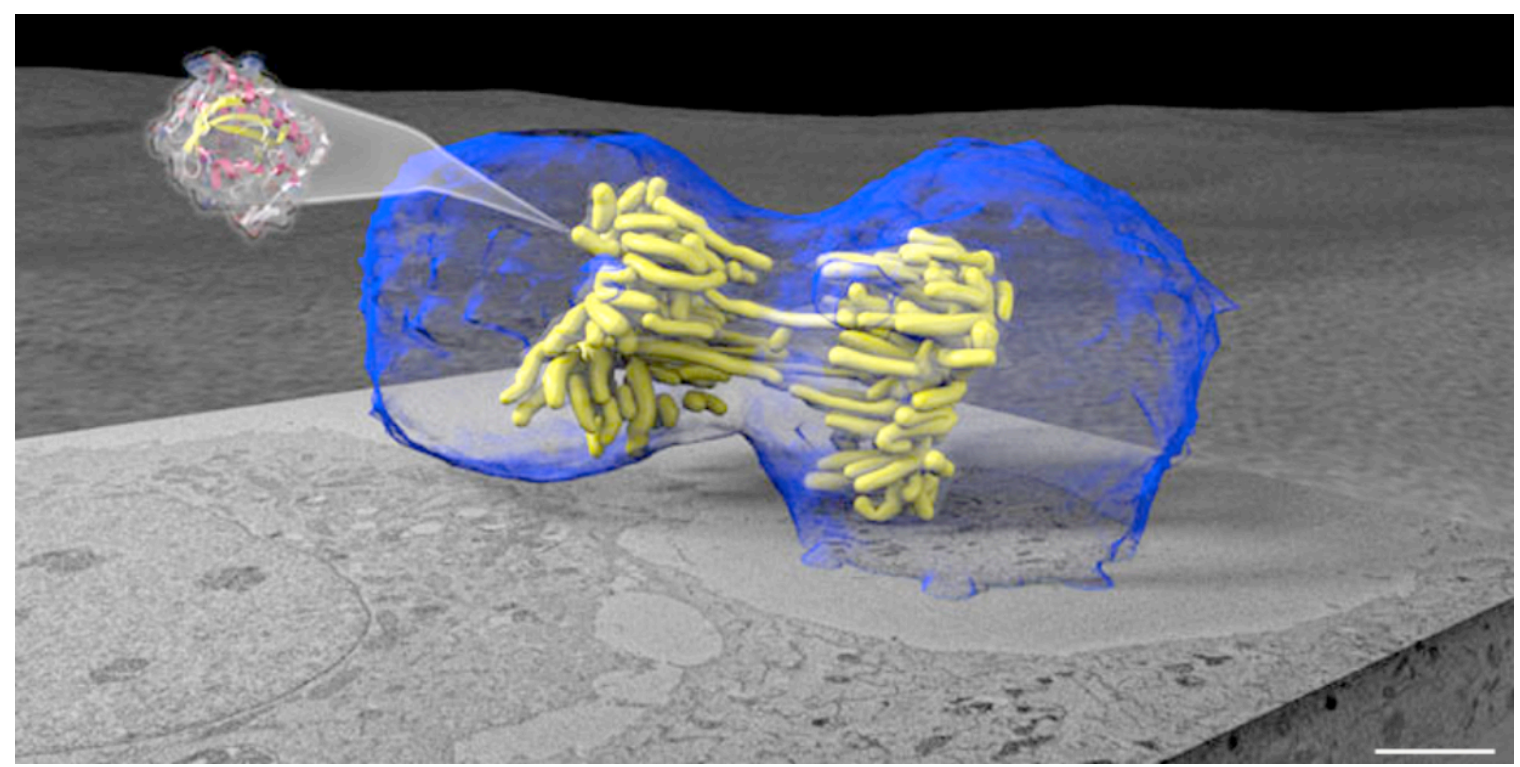

Figure 1. 3-D reconstruction of a dividing cell imaged by SBEM. The cell was transfected to express the fluorescent protein MiniSOG (molecule enlarged and shown in schematic at upper left) fused to Histone $2 \mathrm{~B}$ to label chromosomes (shown in yellow). The outline of the cell is shown in blue. Scale bar $=5$ microns. 